
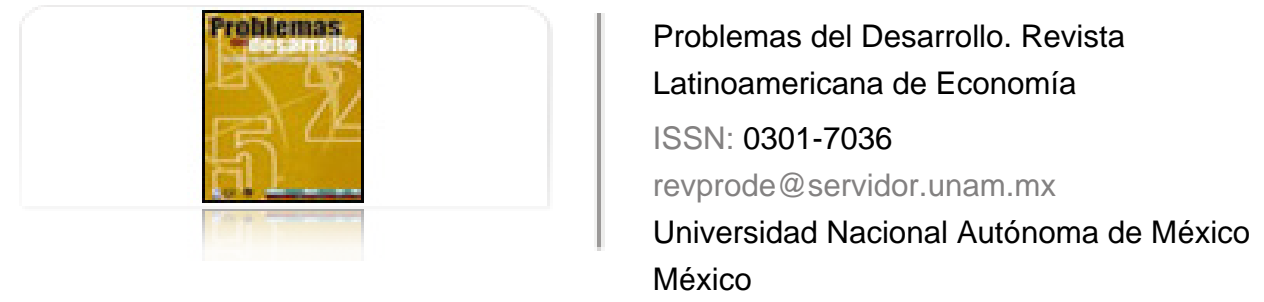

Doppler, Flurina; González, Alma Amalia

El comercio justo: Entre la institucionalización y la confianza

Problemas del Desarrollo. Revista Latinoamericana de Economía, vol. 38, núm. 149, abril-junio, 2007, pp. 181-202

Universidad Nacional Autónoma de México

Distrito Federal, México

Disponible en: http://www.redalyc.org/articulo.oa?id=11820124009

Cómo citar el artículo

- Número completo

- Más información del artículo

Página de la revista en redalyc.org

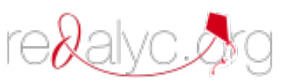

Sistema de Información Científica

Red de Revistas Científicas de América Latina, el Caribe, España y Portugal Proyecto académico sin fines de lucro, desarrollado bajo la iniciativa de acceso abierto 


\section{El COMERCIO JUSTO: \\ ENTRE LA INSTITUCIONALIZACIÓN Y LA CONFIANZA}

\section{Flurina Doppler* Alma Amalia González **}

Fecha de recepción: 19 de agosto de 2006. Fecha de aceptación: 22 de enero de 2007.

\section{Resumen}

En los inicios del comercio justo, ciertas posturas críticas señalaron la limitación de su alcance por tratarse de un nicho de mercado de fácil saturación. Hoy en día demuestra que representa una importante cifra de negocios para las empresas sociales, pero también para los consorcios empresariales. Derivado de ese auge, es necesario crear normas e instituciones que validen su funcionamiento. El trabajo presenta los inicios de la certificación solidaria y el escenario de las organizaciones cafetaleras de Chiapas del comercio justo. Posteriormente, se enfoca a los procesos de certificación, la participación de los productores y en sus respuestas organizativas. Se muestran evidencias que remarcan la importancia de la confianza y los riesgos que la institucionalización representa en el deterioro de los valores simbólicos movilizados.

Palabras clave: mercados alternativos, institucionalización, procesos de certificación, cafeticultura indígena, Chiapas.

* Investigadora especializada en problemas sociales del sector cafetalero y mercados alternativos; en la actualidad, sin adscripción institucional. Egresada del instituto de Antropología Social de la Universidad de Fribourg, Suiza, y maestra en Ciencias por el Centro de Investigaciones en Antropología Social (CIESAS), México. Correo electrónico: flurina.doppler@gmx.ch

* Investigadora del Programa de Investigaciones Multidisciplinarias sobre Mesoamérica y el Sureste, del Instituto de Investigaciones Antropológicas, Universidad Nacional Autónoma de México (Prolmmse IIA UnAM), San Cristóbal de Las Casas, Chiapas, México. Correo electrónico: agonzale@servidor.unam.mx 
Abstract

From its beginnings, certain critical postures have pointed out the limitations of fair trade in its attempt to enter easily saturated market niches. Today, this has been shown to represent an important level of business not only for public limited companies, but also for business consortia. As a result of this impact, rules and institutions should be created to validate its workings. The study documents the beginnings of solidarity certification and the scenario for fair trade coffee-producer organizations in Chiapas. It then focuses on the certification processes, the participation of producers and their organizational responses. Evidence is shown emphasizing the importance of confidence and the risks that institutionalization involves in the deterioration of the mobilization of symbolic values.

Key words: alternative markets, institutionalization, processes of certification, indigenous coffee production, Chiapas.

\section{Résumé}

Dans les débuts du commerce équitable, certaines postures critiques signalaient que sa portée était limitée s'agissant d'un secteur de marché rapidement saturé. Aujourd'hui, il a montré qu'il représente un important chiffre d'affaire pour les organisations non gouvernementales mais aussi pour les entreprises. Du fait de cet essor, il est nécessaire de créer des normes et institutions qui valident son fonctionnement. Cette étude fait état des débuts de la certification solidaire et décrit le cadre des organisations de commerce équitable de café du Chiapas. Elle est ensuite centrée sur les processus de certification, la participation des producteurs et leurs réponses aux problèmes d'organisation. Il est mis en évidence que la confiance joue un rôle important et que l'institutionnalisation peut mettre en péril les valeurs symboliques mises en jeu.

Mots clés: marchés alternatifs, institutionnalisation, processus de certification, culture du café par les Indiens, Chiapas.

\section{Resumo}

Nos inícios do comércio justo, certas posturas críticas assinalaram a limitação de seu alcance por se tratar de um nicho de mercado de fácil saturação. Hoje em dia, já provou que representa uma importante cifra de negócios para as empresas sociais, $e$ também para os consórcios empresariais. Derivado desse auge é necessário criar normas e instituições que validem o seu funcionamento. O trabalho apresenta os inícios da certificação solidária e o panorama das organizações cafeteiras de Chiapas do comércio justo. Posteriormente, enfoca os processos de certificação, a participação dos produtores e suas respostas organizativas. Mostram-se evidências que destacam a importância da confiança e os riscos que a institucionalização representa no deterioro dos valores simbólicos mobilizados.

Palavras-chave: mercados alternativos, institucionalização, processos de certificação, cafeicultura indígena, Chiapas. 


\section{Los inicios de la certificación solidaria}

os inicios del sistema del comercio justo, como lo conocemos hoy en día,
remiten a las acciones emprendidas por la Unión de Comunidades Indígenas
de la Región del Istmo (UCIRI) en el estado de Oaxaca, en el sur de México. Del encuentro de ésta y un asesor con un representante de la organización de cooperación internacional holandesa Solidaridad, a finales de la década de los ochenta, surgió la asociación Max Havelaar. ${ }^{1}$ Los principios de esa institución cuestionan la ayuda a países del Sur, ${ }^{2}$ basada en la desigualdad entre donantes y beneficiarios. Igualmente, para una mejor inserción al mercado, el apoyo a los productores marginados podría ser un medio promisorio para superar la pobreza y promover el desarrollo local. No obstante, más allá de esos planteamientos, para que el comercio equitativo fuera una opción viable para un número significativo de productores, fue necesario ir más allá de las redes de distribución solidaria, ${ }^{3}$ cuya clientela estaba limitada a una minoría de personas concientizadas. Fue preciso introducir los productos de comercio justo en los grandes circuitos de distribución, concretamente en los espacios donde la mayoría de los consumidores hace sus compras (Roozen y Van der Hoff, 2002).

En 1988 se vendió el primer café de la UCIRI con el sello de Max Havelaar en los supermercados de Holanda. Este sello surgió teniendo como principios centrales la promoción entre los consumidores de un comercio que —establecidos los lazos directos entre los pequeños productores y los compradores- pague a los productores un precio justo, el cual se basa - teóricamente- en los costos de producción. Hay un precio mínimo que, desde los inicios de la propuesta del comercio justo y

1 Max Havelaar es el título de un libro famoso del autor holandés Edouard Douwes Dekkers (seudónimo Multatuli), escrito en 1859. Su héroe, Max Havelaar, denuncia la opresión de los pequeños campesinos en la colonia indonesa.

2 Este documento utiliza los términos países del Norte y países del Sur, retomándolos del discurso de las iniciativas de comercio justo. Cabe aclarar que en este trabajo no se propone introducir una discusión sobre las teorías que conceptuan el término desarrollo.

3 Desde los años sesenta ya habían empezado a difundirse (principalmente en Europa y, en menor medida, en Estados Unidos) las llamadas "tiendas del tercer mundo" —ahora "tiendas del mundo"-, operadas por grupos de solidaridad, quienes comercializan de manera directa artesanías y alimentos de pequeños productores del Sur. 
hasta la fecha, no ha sido modificado; sin embargo, se eleva si el café es arábica lavado (121 dólares por 100 libras) y si cuenta con un certificado orgánico (136 dólares).$^{4}$ A dicho precio mínimo se añade un premio social de cinco dólares por 100 libras, considerado para fortalecer la inversión en proyectos de beneficio social colectivo. Tomando en cuenta la limitación de los productores para disponer de dinero en efectivo con el fin de realizar el acopio, ${ }^{5}$ el comercio justo declara como uno de sus condicionamientos a los importadores el pago por adelantado de hasta $60 \%$ de la cosecha. Los beneficios antes enunciados fueron aceptados por los importadores y pequeños torrefactores que participaron en el comienzo de la iniciativa Max Havelaar. Cabe resaltar que el debut del comercio justo de café no puede explicarse sin la participación de esos pequeños torrefactores, ${ }^{6}$ quienes también resisten los embates de su baja competitividad frente a las grandes compañías que manejan el negocio mundial del café, a saber: Philip-Morris, Nestlé, Procter and Gamble y Sara Lee (Pouliquen, 2003). Más adelante analizaremos algunas de las dificultades para el cumplimiento de esos principios.

En correspondencia con los principios del comercio justo, se requirió a los pequeños productores que estuviesen asociados en cooperativas que garantizaran la participación democrática, la aceptación de cualquier productor sin discriminación

4 Los criterios de calidad en el café son extremadamente amplios y complejos; sin embargo, para el caso del comercio justo, se manejan básicamente dos criterios. Por un lado, la variedad y, en segundo término, el lavado del grano. Las variedades que se definen son: Coffea arabica y $C$. canephora. En términos generales, la primera se cultiva en tierras de mayor altitud - entre 900 hasta poco más de $1200 \mathrm{msnm}$-, condiciones geográficas que le confieren mayor calidad organoléptica. La variedad C. canephora, comúnmente conocida como Robusta, se cultiva en tierras de baja altitud; sus rendimientos son superiores al primer tipo, pero sus características gustativas son consideradas de menor calidad. El término de café lavado se utiliza para especificar que los granos han pasado un proceso de beneficio húmedo, en el cual se eliminan las cubiertas seminales y los mucílagos; para el posterior secado al sol, hasta obtener $12 \%$ de humedad.

5 Las organizaciones hacen un acopio de la cosecha, el cual se concreta con la entrega que cada productor hace y el pago que éste recibe como adelanto. El pago total se realiza una vez que los compradores han cubierto las facturas de entrega. Es frecuente que las organizaciones tengan problemas de disponibilidad de fondos para pagar a sus agremiados. Los ingresos por la venta de la cosecha de un ciclo de café permiten, básicamente, dicho pago a los socios y los gastos corrientes; situación que provoca una permanente incertidumbre por la falta de fondos para el acopio. De allí, la importancia que tiene el comercio justo, al ofrecer la opción del pago adelantado.

6 La torrefacción es el proceso final del grano del café - que considera el tostado secreto artesanal - y la preparación de mezclas de diferentes tipos de café. En particular, el café es la bebida que adquiere su embrujo por la mezcla y no precisamente por la exclusividad geográfica de su origen. 
por sus ideas políticas, religiosas o su diferencia étnica. En conjunto, dichas condiciones favorecieron la eliminación de los agentes de intermediación local, los coyotes, lo cual hizo posible que las cooperativas tuviesen mayor margen de ganancia; asimismo, también los importadores, torrefactores y distribuidores se vieron favorecidos por el incremento de sus ventas y, en consecuencia, de sus ganancias (Pouliquen, 2003; Pelupessy y van Tilburg, 1994).

\section{El escenario de las organizaciones chiapanecas del comercio justo}

La cafeticultura mexicana - al igual que en muchos otros países productores- fue una importante actividad generadora de divisas, por lo que tuvo un fuerte control estatal. El Instituto Mexicano del Café (INMECAFE, 1958-1993) fue el órgano que reguló las actividades de financiamiento, asistencia técnica y exportación del grano. Tras el retraimiento de la política de Estado en ese sector y a finales de los años ochenta, el comercio justo fue una de las opciones más viables para la comercialización del producto de organizaciones cafetaleras de México.

Si bien es cierto que el INMECAFE tuvo una política generalizada en todas las zonas cafetaleras del país, habría que resaltar que en las zonas con población indígena sus acciones fueron ejercidas mediante el — también extinto- Instituto Nacional Indigenista (INI), que también difundió criterios tecnológicos de la cafeticultura intensiva. Sin embargo, la inaccesibilidad de las parcelas cafetaleras en la agreste topografía del territorio suscitó que sus acciones de asistencia técnica no llegasen a esos recónditos poblados. Este hecho, que en principio representó una desventaja, después permitió que las zonas cafetaleras indígenas tuvieran una valoración especial. El mantenimiento de complejos sistemas bióticos en el cafetal (Moguel y Toledo, 1999), la no utilización de agroquímicos, el uso de sombra diversificada y el trabajo artesanal para el proceso de beneficiado, ${ }^{7}$ entre otras pecualiaridades, han hecho posible que el café de ciertos grupos étnicos sea un producto diferenciado. Las características antes enunciadas fueron promovidas por las organizaciones cafetaleras indígenas ${ }^{8}$ y por las redes de consumidores que construyeron las bases del

7 El café inmediatamente cosechado es sometido a un proceso de fermentación llamado beneficio húmedo, el cual confiere mayor calidad organoléptica al aromático.

8 La Confederación Nacional de Organizaciones Cafetaleras - CNOC, fundada en 1990, y que agremia a organizaciones de pequeños productores - ha favorecido la sociabilización de las experiencias de participación en el comercio justo. Dicha institución actúa también como negociadora ante el Estado para el otorgamiento de recursos financieros al sector. 
comercio justo y el mercado orgánico de café, en el momento de la liberalización económica del sector.

La UCIRI fue la primera institución que exportó directamente su producción de café en el marco del comercio justo. Sus acciones sirvieron para definir la entrada de otras organizaciones campesinas cafetaleras, pues existían fuertes lazos de cooperación basados en la confianza que, además, fue un rasgo caraterístico de las pioneras (Nigh, 1999).

Al momento del desmantelamiento del INMECAFE (1993), los cafetaleros indígenas no tenían organizaciones consolidadas. Ante la falta de regularización en los permisos de exportación, algunas de éstas comenzaron a exportar mediante contratos gestionados por la UCIRI. Ejemplos concretos de ello son la Unión de Ejidos de La Selva (Indígenas de la Sierra Madre de Motozintla, ISMAM, Sociedad de Solidaridad Social) y la Federación Indígena Ecológica de Chiapas (FIECH) (González, 1999 y Doppler, 2006). Las relaciones de cooperación entre las organizaciones campesinas se han mantenido en diversas formas, por ejemplo, mediante cursos de capacitación (impartidos de una institución a otra), ${ }^{9}$ asesoría específica o consejos en un sentido vasto.

Las organizaciones campesinas han privilegiado la cooperación entre sus agremiados. Tal medida está asociada con una visión del desarrollo promovida por líderes y asesores, misma que se inscribe en una discusión de envergadura global. En la medida en que haya capacidad para transformar sus propios espacios de acción, los pequeños productores pueden ser capaces de intervenir en otros ámbitos de la toma de decisiones. ${ }^{10}$ En este caso en particular, su desarrollo estaría en función de su capacidad para participar en la construcción de las reglas del juego del comercio justo (Doppler, 2006; González, 1999).

No obstante que la promoción de cooperación interna es sin duda un medio para el desarrollo local, la consolidación del comercio justo tiende a ser evaluada en tér-

9 Cada año la UCIRI recibe a jóvenes de diversas organizaciones campesinas indígenas para que se capaciten en su centro de formación. A esos cursos han asistido los hijos de productores de organizaciones chiapanecas del comercio justo (ISMAM, La Selva, Tiemelonla nich k'lum, Tzeltal Tzotzil, Productores Agropecuarios de la Selva Lacandona) (http://www.uciri.org/ espanol/proyectos/cec/cec_info.htm, página del Centro de Educación Campesina de la UCIRI.

10 La discusión teórica y política acerca de la participación en la toma de decisiones nos remite al concepto empowermet (véanse, por ejemplo, Cheater, 1999; Freire, 1977 y Friedmann, 1992). No se pretende aquí desarrollar la evolución y discusiones en torno de tal término, sino básicamente retomarlo para explicar las acciones de las organizaciones campesinas en la construcción del comercio justo. 
minos mercantiles. El acceso al mercado implica obtener ingresos económicos directos, de allí que, más allá de las redes de cooperación local entre organizaciones, el comercio justo impone una competencia para apropiarse de una mayor parte de ese segmento del mercado. Esa competencia conlleva a la necesidad de regular el acceso a nuevas organizaciones, a fin de evitar la saturación de la demanda. ${ }^{11}$ Una de las formas en que se ejerce tal regulación es mediante el establecimiento de reglas y criterios para obtener el sello o la acreditación de las iniciativas de comercio justo en los países consumidores, tema sobre el cual se profundizará más adelante.

\section{Percepciones diferentes}

El hecho de convertir una empresa social orientada al mercado internacional de café demanda de los pequeños productores un fuerte proceso de aprendizaje, para lo cual las organizaciones pioneras contaron con el apoyo, acompañamiento y asesoría de las personas involucradas en el comercio justo. Particularmente, fueron miembros de distintas iglesias quienes promovieron en los países del Norte el consumo solidario y en los países del Sur actuaron como asesores de un proceso de reconversión productivo y comercial, impregnado de un marcado idealismo. Desde los comienzos de dicha propuesta, las organizaciones cafetaleras manifestaron una conciencia por la conservación de los recursos naturales, lo cual les ha llevado a apegarse a las técnicas y concepciones holísticas del movimiento de agricultura orgánica (González y Nigh, 2005).

Quienes participamos en los proyectos que tienen que ver con la agricultura orgánica somos un tanto soñadores. Tal vez sí sea cierto que pensamos en una utopía. Sin embargo, estamos convencidos de esto y por ello seguimos el camino. Tenemos muchas desventajas. Por lo que se refiere al café, que es el cultivo donde más agricultura orgánica se hace, nos enfrentamos al mercado. Competir en el mercado internacional con un producto diferenciado no implica que estemos exentos de las reglas que se imponen en la fijación de precios en la bolsa de valores [...] En realidad no creo que los productores estén ganando realmente más; sin embargo, estamos convencidos del sacrificio que debe hacerse para recuperar la tierra que permitirá la vida de las generaciones futuras. ${ }^{12}$

11 El sobreprecio del comercio justo está basado en la no saturación de la demanda del nicho de mercado (González et al., 2003).

12 Entrevista realizada en 1999 a un clérigo asesor de una organización cafetalera indígena que exporta en el marco del comercio justo. 
Los productores de organizaciones como ISMAM y La Selva recuerdan cómo fueron visitados por los consumidores, generalmente miembros de grupos parroquiales, quienes actuaron de manera altruista en las campañas de promoción y concientización del comercio justo (González, 2002). Así narra don Arturo al recordar los comentarios de damas holandesas que los visitaron en sus comunidades, para conocer directamente en qué forma contribuía el sobreprecio.

Las cosas eran más parejas antes de que estuviese FLo, porque así como nos tocó ir a Europa, también las señoras de las iglesias vinieron a conocer nuestras casas. Cuando ellas vinieron, se dieron cuenta de todas nuestras necesidades, ya ni siquiera se preocupaban por preguntar, en qué habíamos ocupado el sobreprecio que ellas habían pagado por el café, sino que decían: “qué más podemos hacer?”13

Para los dirigentes de las organizaciones chiapanecas que han participado inicialmente en el comercio justo, el papel de Max Havelaar ha sido claro. Ellos saben que esa iniciativa no se dedica a comerciar, sino que su intervención les permite obtener un sobreprecio para su café. Sin embargo, el significado de mercado justo tiene diversas percepciones. Algunas organizaciones como ISMAM $^{14}$ declaran que el comercio justo es sólo un elemento que facilita el desenvolvimiento de su experiencia en el mercado internacional, pues saben que la demanda no crece al ritmo necesario para absorber la oferta.

La percepción de los productores sobre el comercio justo es bastante relativa, ya que el nivel de información de los agremiados suele ser extremadamente diferente. Por un lado, existen productores que, habiendo ocupado puestos directivos en la organización, poseen más datos, pero son un grupo muy reducido. La mayoría de los socios de una organización de productores no han aprendido el objetivo del comercio justo y en muchos casos ni siquiera saben que su institución participa en ese nicho de mercado. En algunos casos, los productores perciben el sobreprecio como compensación por sus condiciones de pobreza (González, 2002).

En parte, el desconocimiento de cómo funciona el comercio justo se debe a la complejidad del sistema, el cual, en el caso del café, además, está estrechamente vinculado con la percepción de buena calidad a partir de la certificación orgánica. La falta de comprensión del mercado solidario y de los requisitos del mercado de exportación en general debilita dicho comercio como propuesta de desarrollo local.

13 Comentario de don Arturo, líder fundador de La Selva (González, 2002).

14 Entrevistas realizadas en 1999 y 2002 a asesores técnicos de ISMAM. 


\section{La institucionalización del comercio justo: FLO-International}

Como bien señalan diversos autores (Raynolds, 2002; Rice, 2001 y Tallontire, 2000) el éxito del comercio justo fue probablemente el motivo de su paradoja. En este apartado haremos un esfuerzo para aclararla.

De 1988 a 1997, el campo social de dicho comercio se caracterizó por la proliferación de iniciativas nacionales en los países consumidores que construyeron sus propios sistemas. En algunos casos, lo hicieron a partir de la imagen de Max Havelaar y, en otros, se hizo creando nuevos sellos, los cuales permitieran identificar un producto que, a diferencia de la marca, no compromete en aspectos fiscales ante el Estado.

Con el objetivo de dar coherencia a los postulados y criterios de las diferentes iniciativas nacionales de comercio justo, en 1997 se optó por integrar un buró que agremiara a la mayor parte de éstas. La medida también se previó como mecanismo para centrar esfuerzos — sobre todo en cuanto a mercadotecnia - que permitan incrementar el volumen de ventas. Fue así como se creó la Fairtrade Labeling Organizations (FLo-International), que tiene su sede en Bonn, Alemania. En la actualidad esa organización integra:

- 20 iniciativas nacionales

- 531 organizaciones de productores certificadas, las cuales representan más de un millón familias de productores y trabajadores en 50 países en África, Asia y Latinoamérica.

- 667 comerciantes registrados: exportadores, importadores, procesadores y manufactureros provenientes de 50 países esparcidos por todo el mundo. ${ }^{15}$

- 550 personas que tienen la licencia, autorizada por las organizaciones afiliadas de la FLo - las Iniciativas Nacionales_- para utilizar el sello de comercio justo en los productos que venden a los consumidores de 20 países en Europa, América del Norte, Japón, México y Australia y Nueva Zelanda. ${ }^{16}$

Este nuevo organismo integrador del comercio justo representa la institucionalización en su dimensión global. Dicha institucionalización se ha concretado con la definición de reglas de participación y mecanismos de sanción que se orientan a

15 Véase: http://www.fairtrade.net/sites/impact/spanish/facts.html, abril de 2006.

16 Véase: $h t t p: / / W w w . f a i r t r a d e . n e t / s i t e s / i m p a c t / s p a n i s h / f a c t s . h t m 1$, junio de 2005. No existen datos para 2006. 
garantizar al consumidor la incorporación de los valores de solidaridad. El reto de la FLo-International ha consistido en ampliar el beneficio de comercio justo al mayor número de organizaciones de pequeños productores. Para ello se establecen dos estrategias: $a$ ) incrementar el volumen de ventas de los productos del comercio justo y $b$ ) diversificar los productos. Esas acciones se cumplen mediante grandes campañas de mercadotecnia en los países consumidores, sobre todo la información al consumidor y la utilización de canales masivos de comercialización. Otra estrategia adoptada por FLo-International para incidir en el incremento del volumen de ventas se ha basado en la apertura a la participación de compañías internacionales, las cuales se han visto atraídas por el acelerado incremento de ventas del nicho de mercado (Renard, 2005).

Con la entrada de esas grandes empresas al sistema y con la venta de los productos en los supermercados — es decir, con el mainstreaming de los productos justosse logró aumentar de manera sustancial su participación en el mercado. ${ }^{17} \mathrm{Sin}$ embargo, mientras que al inicio el comercio justo funcionaba - principalmente apelando a la solidaridad de los consumidores con los pequeños productores-, los esfuerzos de posicionamiento en el mercado convencional implicaron que la calidad de los productos llegara a ser un factor cada vez más importante. Con la colocación del producto solidario en los anaqueles como un café más, al lado de una amplia oferta de diferente calidad y origen, el producto justo compite por la preferencia del consumidor. Así pues, el planteamiento original del comercio justo por construir una forma de comercializar alternativa al sistema del mercado global pierde vigencia. Además, "el incremento en el volumen de ventas implicaba que participaría un mayor número de agentes en la comercialización en un ámbito más despersonalizado, con riesgos de corromper los valores de solidaridad social que se movilizaban" (González, 2003:154) y así debilitar la confianza en el sistema. Eso mismo introduce la necesidad de establecer reglas de supervisión y sanción, a fin de evitar la entrada del pasajero invisible o gorrón ${ }^{18}$ que, sin compartir los valores

17 Las ventas de los productos certificados por la FLO International crecieron entre $1997 \mathrm{y}$ 1998 de 25972 a 28913 toneladas; es decir, aumentaron 11.3\%; entre 2003 y 2004 el sector experimentó un crecimiento de $56 \%$. Véase www.fairtrade.net/sites/impact/spanish/ facts.html, abril de 2006. Para más cifras sobre el desarrollo del mercado justo, véase Raynolds (2002), Murray, Raynolds y Taylor (2006), así como las estadísticas en la página de la Asociación Europea del Comercio Justo (EFTA, por sus siglas en inglés) (http:// Www.european fair trade association. org/Efta/Doc/stats2004.pdf).

18 El gorrón puede estar en el país consumidor - representado por los agentes que intervienen en la importación, torrefacción y distribución del producto-, pero también pueden ser 
éticos en juego, se beneficia con el sobreprecio al cual puede llegar con la imagen del comercio justo.

\section{La confianza}

El comercio justo se basa en la confianza del consumidor — quien sustenta el sobreprecio-, pues sólo está dispuesto a pagar dicho sobreprecio si tiene la seguridad de que éste realmente llegará a los productores marginados. Esa confianza se basa en un proceso sistémico altamente abstracto, típico de las sociedades posmodernas. ${ }^{19}$ El consumidor deja de cuestionarse por lo que pasa detrás de su compra y delega en el sistema la responsabilidad de definir aquello que remite a la solidaridad social y desarrollo sustentable de las organizaciones campesinas de países del Sur que participan en el comercio justo.

Para depositar la confianza en un sistema se precisa que éste ofrezca el respaldo de un conjunto de normas y procedimientos emitidos por expertos, además, que exista una posición imparcial para actuar en caso de ameritarse una corrección o sanción ante el incumplimiento de la norma. En el caso de los sistemas complejos, la confianza se opera mediante procesos de certificación — para el caso, la certificación solidaria.

La certificación del comercio justo facilita que el consumidor se libere del aspecto negativo que para él tienen sus actitudes consumistas. Ahora es factible consumir con responsabilidad y dejar atrás las posiciones de rechazo al mercado como agente regulador. Finalmente, el mercado ha logrado incorporar los valores éticos y movilizarlos como mercancía para satisfacer la moral de los consumidores (Lipovetsky, 2005; González et al., 2003).

Hasta este punto, la confianza se ha limitado a aquella que el consumidor deposita en el sistema de los sellos o símbolos respaldados por la certificación solidaria. Sin embargo, en un sistema complejo, se extiende a todos los agentes que intervienen en el dispositivo. Para ir afinando, acotemos lo que se entiende por confianza. Tengo confianza en aquellos a quienes conozco porque estoy seguro que ellos actuarán conmigo de la misma manera en que yo lo haría con ellos. Tener confianza

organizaciones de productores que no cumplen con los criterios promovidos por el beneficio social del productor.

19 Entendemos por sociedades posmodernas aquellas que han perdido la fe en la razón (Lipovetsky, 2005). 
en algo o alguien implica que conocemos su conducta pasada y tenemos certidumbre en lo que hará en determinadas situaciones futuras (Luhmann, 1996).

El comercio justo facilita al consumidor su acto de confianza, pero, ¿hasta qué punto permite que los demás agentes que intervienen la tengan entre sí? Aquí se introduce una serie de consideraciones poco abordadas, ya que el objetivo central para construir la confianza se ha orientado al consumidor. Sin embargo, para que el comercio justo funcione, se necesita que haya confianza entre los productores miembros de una organización. Igualmente se requiere la confianza mutua entre los productores y los compradores-importadores, sin la cual no sería posible reconcretar el acto de venta que permite la exportación. En un sentido más amplio, se necesita la confianza en el buen funcionamiento del engranaje del sistema FLo-International no sólo de parte de los consumidores, sino también del resto de los agentes involucrados.

Al tenor del crecimiento y complejidad del comercio justo, ha sido necesario el fortalecimiento de los mecanismos que validan la confianza en el dispositivo. Como hemos señalado anteriormente, en ese sentido, los esfuerzos se han enfocado principalmente en los consumidores. Así pues, el comercio justo deja su posición de alternativo para remitirse, una vez más, a las reglas utilizadas por el mercado global, como corresponde al caso: los mecanismos de certificación.

\section{Certificando la certificación}

La reglamentación del mercado internacional demanda que las agencias - ya sean privadas o asociaciones sin fines de lucro- que se dediquen a certificar un producto o proceso deben dar confianza en aquello que dicen ofrecer (Touraine, 1995). Para lograrlo, en primer lugar, las agencias deben demostrar su imparcialidad y su sistematización para llevar a cabo los procesos de certificación y auditorías de trayectoria comercial.

La vorágine por certificar la calidad de un producto o servicio se ha hecho sentir también en el comercio justo. Resultado de ello fue la determinación de establecer un agente independiente que realizara el proceso para validar la incorporación de valores de solidaridad social. Es así como nació la Flo-Cert en 2002. Una vez creado el organismo certificador, se necesitó ratificar su validez de acuerdo con las normas establecidas por el mercado global (Mangematin, 1999). Previo a ello, el primer paso fue la acreditación o certificación por parte de la entidad suiza International Standards Organization (ISO), en particular la norma 65, correspon- 


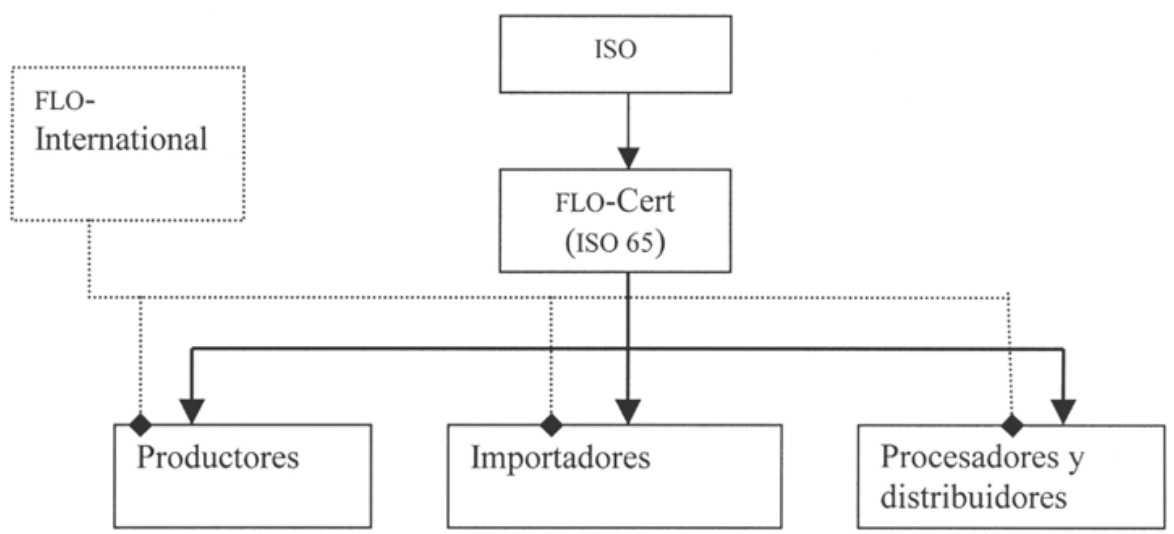

Gráfica 1. Esquema del proceso de certificación de la FLo-Cert bajo la norma ISO 65.

diente a "Exigencias generales relativas a los organismos dedicados a la certificación de productos". ${ }^{20} \mathrm{Al}$ respecto, la FLO considera que:

El motivo principal del establecimiento de Flo-Cert como una sociedad limitada es hacer más transparente la certificación y las operaciones de auditoría comercial. La creación de FLo-Cert amplía la autonomía de sus decisiones sobre certificación y registro de comerciantes, y facilita el cumplimiento con los Estándares iso para Organismos de Certificación (ISO 65).

Las medidas hasta aquí adoptadas por la FLo-International han servido para consolidar la propuesta de comercio justo como un atractivo nicho del mercado global. En la actualidad no existe duda de las ventajas económicas por participar en ese tipo de comercio. Sin embargo, asentar la confianza en un proceso de certificación sofisticado "que exige niveles de conocimientos técnicos, sistemas de gestión y de administración, medios financieros, que no siempre son dados en los países en desarrollo" (Speich, 2001:285), debilita la posición de los pequeños productores en el sistema. Mientras que en los primeros años el comercio justo se caracterizaba por contactos personalizados — con el afán de cumplir las exigencias de las reglas institucionales para generar confianza-, ahora el sistema se ha hecho cada vez más abstracto (véase gráfica 1), lo cual lo hace impersonal para las organizaciones de productores.

El actual esquema de certificación solidario de la FLO-Cert pretende validar una posición de imparcialidad con base en su autosuficiencia financiera. El antiguo

20 Véase $h t t p: / / W w w . i s o . o r g / i s o / f r / C a t a l o g u e D e t a i l P a g e . C a t a l o g u e D e t a i l ? C S N U M B E R=$ $26796 \& I C S 1=3 \& I C S 2=120 \& I C S 3=20$, abril de 2006. 
sistema de las iniciativas nacionales y más tarde de la FLo-International se caracterizó por no fijar ningún pago a los productores en el dispositivo al otorgar un voto de confianza o acreditación. Esa política se planteaba a partir de la concientización del hecho de que muchos pequeños productores practicaban una forma de agricultura orgánica, pero que no podían tener acceso a los sobreprecios del mercado orgánico por los altos costos de la certificación. ${ }^{21}$ El financiamiento de las actividades de acreditación recayó en los concesionarios que pagaban a las iniciativas nacionales por el uso del sello y el precio del producto que adquiere el consumidor solidario.

Sin embargo, la profesionalización e institucionalización del dispositivo ha traído diversos cambios para las organizaciones de productores en su relación con el comercio justo.

A partir de la creación de la FLo-Cert se pretende tener mayor autonomía financiera, a fin de dar transparencia e imparcialidad a los procesos de certificación y auditoría de trayectoria. ${ }^{22}$ Esta medida ha obligado a ejercer mayor presión en las cuotas pagadas por los miembros del dispositivo, en particular para las organizaciones campesinas, contraviniendo el planteamiento original de no incrementar los costos de producción.

La exigencia se plantea crítica para las organizaciones de productores, ya que la certificación solidaria deviene un nuevo costo de producción. En la actualidad —después de una mayor polémica y ajustes- ${ }^{23}$ se han fijado tarifas por conceptos de inscripción al dispositivo, de inspección inicial y de renovación anual del certificado.

La definición de las cuotas es uno de los puntos que mayor controversia ha desencadenado entre las organizaciones de productores. En primer lugar, porque poseer el certificado de comercio justo no les garantiza tener un comprador. De hecho, un requisito preliminar para obtener la certificación estipula que las organizaciones de productores deben tener experiencia previa en la exportación o haber identificado un comprador potencial - de igual manera, certificado por comercio justo.

Las organizaciones de productores reconocen la importancia de hacer aportaciones financieras para el funcionamiento de la fLo-Cert. Aceptan y reconocen la

21 Www.fairtrade.net, agosto de 2001.

22 La auditoría de trayectoria se refiere al seguimiento documental de un producto. Para el caso de los agroalimentarios, el control permite verificar el origen desde la parcela del productor hasta las presentaciones individuales en los centros de venta. Esa medida tiende a evitar las situaciones de fraude por el manejo indebido del sello de la certificación, ya que permite cotejar el flujo del producto.

23 Véase www.boncafe.org/cat/campanya\%20e1\%20bon\%20cafe_archivos/pdf/boncafe 10.pdf o Doppler, 2006 para mayor información sobre estas polémicas. 
ventaja de un nicho de mercado que ofrece un sobreprecio. Sin embargo, las organizaciones de cafetaleros aseveran que el funcionamiento del nicho de mercado de la FLo no está preparado para resolver una situación de precios altos de café, ya que en este caso el diferencial de precios tiende a disminuir e incluso a igualarse. ${ }^{24}$ Diversos autores han señalado esa debilidad del comercio justo de café y destacan que el pago de un sobreprecio no resuelve el problema de la distribución del margen de ganancia entre los actores que participan (González et al., 2003; Muradian y Pelupessy, 2005).

Otras polémicas que se han dado en los últimos años entre los agentes que participan en el sistema flo son: la discusión sobre la viabilidad de certificar plantaciones privadas de café, la propuesta de la Flo de diferenciar los precios mínimos según regiones, la costosa definición de nuevos estándares para productos que benefician a un reducido grupo de productores y la estructura para la toma de decisiones en dicho organismo. Esos señalamientos dejan en evidencia la fragilidad de los sistemas sociales complejos, por lo cual es necesario reforzar las formas de participación que redundan en la construcción de las relaciones de confianza.

\section{Organizaciones campesinas y su representación en la FLO}

El interés de este apartado es discutir la participación y representatividad de las organizaciones de pequeños productores en la toma de decisiones prioritarias, punto que, como se señaló anteriormente, constituye una de las polémicas centrales en la relación de la FLo con los productores.

El deslinde de funciones entre la FLo-Cert y la FLo-International deja a la primera la responsabilidad básica del proceso de certificación e inspección; en tanto que la segunda es responsable de la definición de estándares, financiamiento y enlace directo con los países productores. A diferencia de la Flo-Cert, que se constituyó como empresa, la FLo-International es una asociación sin fines de lucro. Ambas coinciden en un consejo de dirección en el que participan el director general de cada instancia, quienes actúan paralela e independientemente. Asimismo, también los representantes de Foro FLo y la Asamblea de Miembros, integra-

24 Esta situación se presenta para el caso de la miel, producto que llega a alcanzar precios más altos en el mercado nacional que aquellos ofrecidos en el internacional de comercio justo. Trabajo de campo de seguimiento desde 1992 hasta la fecha, con la Sociedad Cooperativa Productores Agropecuarios de La Selva Lacandona, en Ocosingo, Chiapas. 


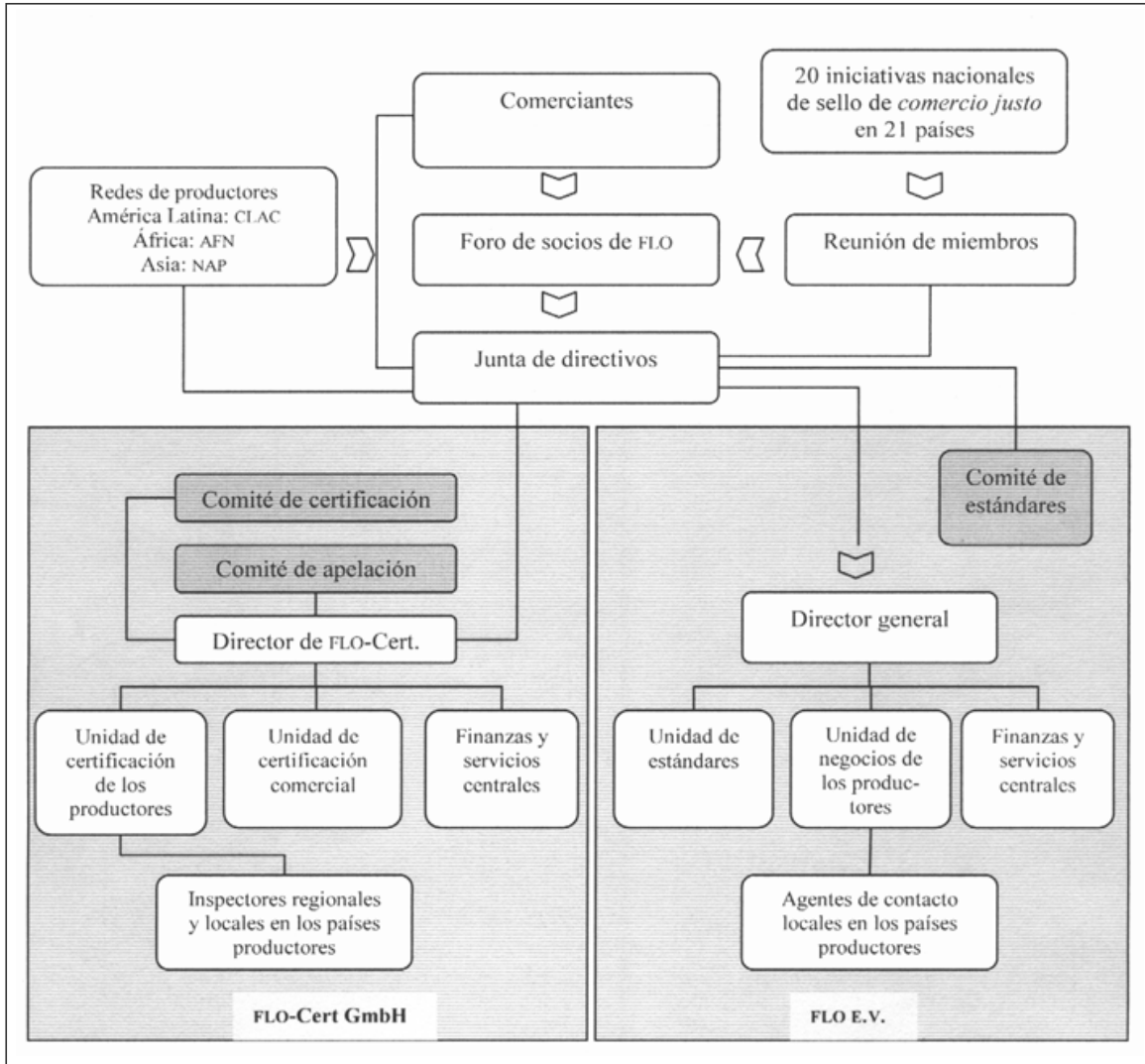

Gráfica 2. Estructura de la FLO (http://www.fairtrade.net/structure.html? $\& L=l_{2}$ agosto de 2006, traducción de las autoras.

da por representantes de las Iniciativas Nacionales, organismo responsable del uso del sello comercial.

La información de la página de la FLo disponible en Internet es bastante dispersa y en ocasiones vaga y contradictoria, aunado a esto está el hecho de que las versiones de los textos en inglés y español son frecuentemente disímiles. ${ }^{25}$ Por lo que

25 Así, por ejemplo, se menciona en una parte de la página de Internet de la flo que los productores "pagan por la certificación y adicionalmente abonan una cuota anual que depende del volumen de sus ventas bajo condiciones de comercio justo" (www.fairtrade.net) sites/aboutflo/spanish/financed.html), mientras que en otro lugar se señala que "a partir de 2006 no se emitirá ninguna factura más por cuota anual o por volumen" (www.fairtrade. net/sites/certification/Fee_2006_Spanish.html). Partes importantes de la página sólo están disponibles en inglés, lo cual hace inaccesible la información a los productores. 


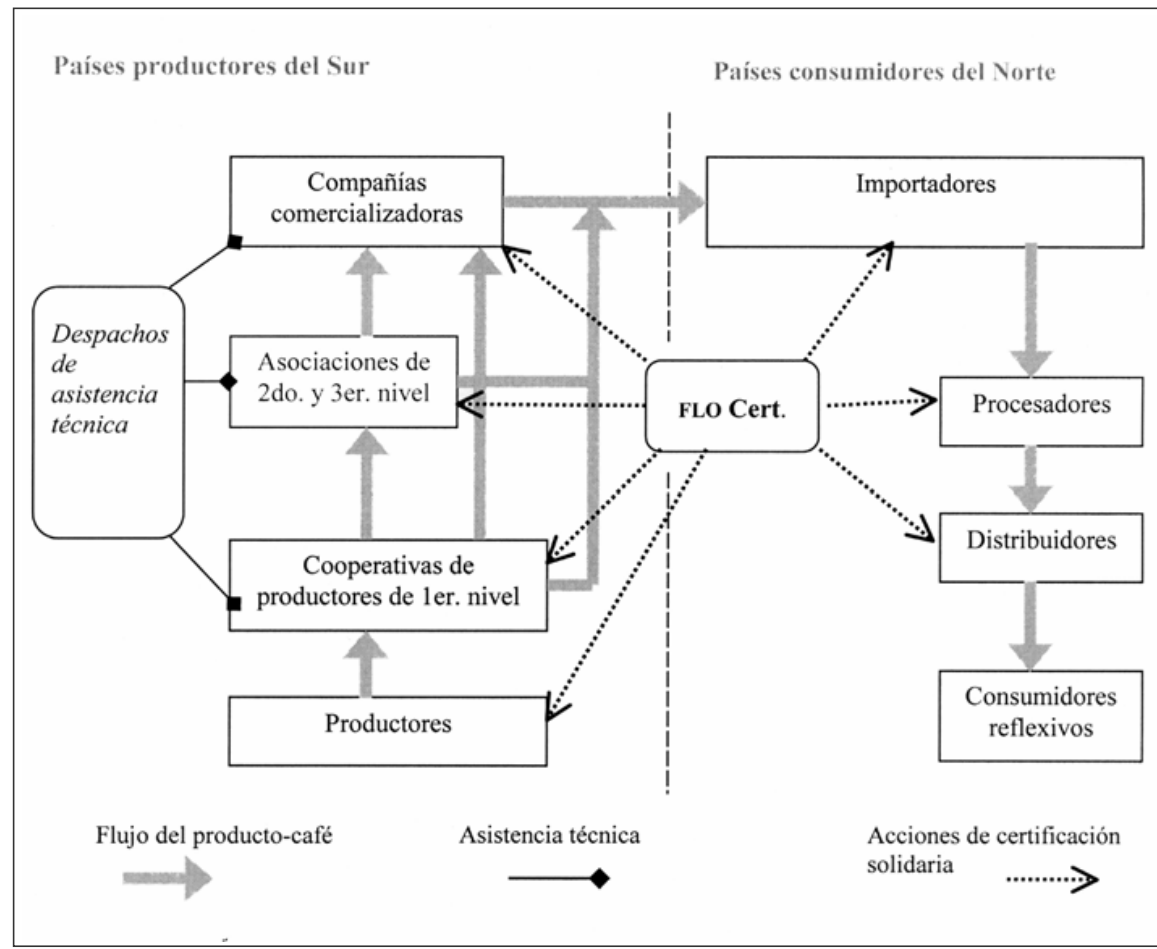

Gráfica 3. Proceso de certificación solidaria de la FLo-Cert.

respecta a la participación de los representantes de los productores en el sistema, la información proporcionada por la FLo en su página web acusa de su integración al Foro Flo. No se proporcionan mayores detalles, pero la gráfica 2 indica que los productores participantes en la estructura de FLo-Cert lo hacen por medio de los Comités de Certificación y de Apelación; mientras que se incorporan a la FLOInternational, lo hacen en el Comité de Estándares. Sin embargo, más allá de lo presentado en los sitios web, ciertamente, en la práctica los espacios de participación de los productores son sumamente limitados.

Para el caso de México, los productores eligen un representante en la asamblea general de comercio justo, que agremia al total de las organizaciones cafetaleras participantes. No obstante, la participación en la toma de decisiones es muy restringida y, además, la estructura actual no garantiza la transmisión de la información a los productores de base, problema que tienen muy bien identificado en diversos ámbitos. Por un lado, se utilizan términos comerciales y financieros con los cuales 
no están familiarizados los productores; otro aspecto igualmente vulnerable es la falta de continuidad en el proceso de participación, ya que las exigencias de la cotidianidad de las organizaciones campesinas les impide dar continuidad a la representación de sus comisionados en las sesiones informativas. Fallas de ese tipo debilitan al comercio justo como propuesta de desarrollo local, entendido éste como participación en la toma de decisiones.

\section{Respuestas organizativas ante las certificaciones}

Los apartados anteriores de este trabajo muestran la complejidad institucional del comercio justo y señalan, además, las desventajas para las organizaciones de productores. En éste se discute acerca de la respuesta y recomposición de las organizaciones locales, con el objetivo de adaptarse al vertiginoso proceso de institucionalización del comercio justo (véase gráfica 3).

Como se dijo en párrafos preliminares, especialmente en el caso del café, la acreditación del comercio justo ha ido a la par de la certificación orgánica. Ambos mercados imponen un incremento en los costos administrativos marcados por las certificaciones y el proceso mismo de exportación. En consecuencia, una estrategia comúnmente adoptada por las organizaciones campesinas para reducir ese impacto negativo es el incremento de su membresía, a fin de aumentar el volumen de producción, cayendo en la consabida lógica de reducción de costos con base en el incremento de volúmenes.

En ese sentido, las organizaciones campesinas han readaptado sus estructuras organizacionales, especialmente formando asociaciones de segundo y tercer niveles. En términos generales, las asociaciones de productores adoptan diversas figuras jurídicas, en función de la conveniencia que éstas ofrezcan para tener acceso a fuentes crediticias gubernamentales o de la banca comercial y en los trámites para la exportación. En cualquier nivel asociativo la estructura organizativa ratifica el funcionamiento democrático: la toma de decisiones recae en la asamblea general y, además, delega las decisiones de tipo comercial a la mesa directiva y al consejo de vigilancia, aquellas que conciernen al cumplimiento de reglas internas.

El procedimiento de la acreditación en el comercio justo se lleva a cabo para la organización de máximo nivel asociativo. Dado el caso de la integración de nuevas organizaciones, éstas son condicionadas a cumplir sus criterios, asegurando así el mantenimiento de la certificación solidaria para la organización de mayor nivel. La concertación de contratos comerciales con importadores de comercio justo no está 
regida por un único procedimiento, aunque, por lo general, es la figura de mayor nivel asociativo quien concreta las operaciones comerciales.

Una situación distinta a la anterior se plantea cuando las organizaciones de productores de primer nivel o de mayor integración obtienen la certificación solidaria de manera directa, pero no realizan directamente la exportación, sino que recurren a los servicios de compañías comercializadoras. Éstas son reconocidas por el comercio justo y para ellas existen normas y criterios específicos que deben cumplir para obtener su acreditación. En México, ostentan frecuentemente la figura jurídica de sociedad civil o sociedad anónima, mediante las cuales llevan a cabo la identificación de clientes, concertación de contratos y preparación de la documentación para la exportación.

El funcionamiento del sistema de comercio justo pareciera estar promoviendo la consolidación de organizaciones que delegan la toma de decisiones en grupos de expertos, lo cual debilita fuertemente la participación directa de los individuos en la toma de decisiones y en la construcción de los procesos de aprendizaje. En ese sentido, cabe señalar otra figura asociativa conocida como despacho, que se especializa sobre todo en aspectos técnico-productivos que permitirán cumplir los criterios para las certificaciones de sellos como la producción orgánica o el café de sombra. ${ }^{26}$ Dicho actor también es clave en la negociación de fondos crediticios provenientes de la banca comercial o de programas gubernamentales (Aguilar, 2005; González y Nigh, 2005) y destinados principalmente a fomentar la exportación de cultivos con certificado orgánico.

Los despachos y las compañías comercializadoras están altamente profesionalizadas y si bien es cierto que son entes independientes, en la práctica existe una estrecha relación entre ambas, justificada por el objetivo de facilitar el funcionamiento de las organizaciones como empresas sociales.

La exposición anterior hace evidente el complejo entramado de figuras asociativas con las cuales deben relacionarse las organizaciones campesinas para tener acceso al comercio justo. Esta orquestación sirve de base para la institucionalización de dicho comercio en el ámbito nacional. La eficiencia y el manejo transparente de las diversas figuras que intervienen es un punto que no necesariamente está en tela de

26 Se trata de un café proveniente de plantaciones que, en particular, cumplen los criterios que validan la biodiversidad de las especies utilizadas como sombra y cuya presencia garantiza la supervivencia de determinadas especies de aves migratorias. La Fundación Smithsonian y Rainforest Alliance son dos de las instancias que ofrecen la certificación de ese sello (González y Nigh, 2005). 
juicio. Sin embargo, lo que sí amerita una reflexión es el efecto que el comercio justo tiene como opción de desarrollo local, entendido como participación en los procesos en la toma de decisiones de las organizaciones campesinas.

\section{Conclusiones}

La institucionalización del comercio justo se ha planteado como una estrategia que reafirma la confianza del consumidor. Sus procedimientos han exigido el establecimiento de normas y procedimientos complejos y abstractos que han generado un distanciamiento entre la estructura central de la FLO y las organizaciones campesinas.

El reto social estriba en mantener la confianza entre los consumidores y los demás agentes comerciales que intervienen en el dispositivo, sin perder de vista que debe estar fundado en mecanismos de información que estrechen la comprensión de las condiciones sociales de los pequeños productores en los países del Sur. Es prioritario promover la reflexión acerca del equilibrio que debe existir entre el ímpetu por alcanzar mayor eficiencia comercial y los postulados que dieron origen al comercio justo. La meta es crear opciones que promuevan el desarrollo local de las sociedades rurales en los países del Sur.

Por otro lado, cabe resaltar que la capacidad de generación de propuestas concretas de parte de las organizaciones campesinas es bastante limitada. La estructura de participación y representación que imponen los mercados de exportación, sean de comercio justo o no, se apoyan en formas jerárquicas con sentido empresarial. La falta de comprensión del dispositivo por parte de los pequeños productores y las condiciones establecidas por factores estructurales de las economías campesinas de países del Sur dificultan la elaboración de propuestas concretas que redunden en la apropiación de dicho comercio como opción de desarrollo.

Finalmente, el comercio justo ha iniciado un proceso de institucionalización que sin duda cobrará mayor fuerza, en la medida en que decidan intervenir las instancias estatales de los países consumidores — tal y como ha sucedido con la agricultura orgánica-. Sin embargo, sus particularidades y, en general, cualquier tipo de certificación sobre normas de conducta adoptadas voluntariamente plantean controversias aún sin resolver en el marco de la normatividad de la Organización Internacional del Comercio. 
Bibliografía

Aguilar Pinto, Emma, Una aproximación antropológica a las organizaciones de agricultura orgánica en la micro región norte: procesos de intermediación económica y liderazgo, tesis de maestría, San Cristóbal de las Casas, CIESAS, 2005.

Cheater, Angela, "Power in postmodern era", en A. Cheater (editor), The Anthropology of Power. Empowerment and Disempowerment in Changing Structures, Londres, Routledge, 1999, pp. 1-12.

Doppler, Flurina, "Los cafetaleros del Rincón y su participación en los mercados alternativos. La configuración de un nuevo campo social", tesis de maestría, San Cristóbal de las Casas, CIESAS, 2006.

European Fair Trade Association (EFTA), http:// www.european-fair-trade-association.org/ Efta/Doc/stats2004.pdf

Fairtrade Labelling Organisations International (FLO), www.fairtrade.net, 2006

Freire, Paulo, Cartas a Guinea-Bissau. Apuntes de una experiencia pedagógica en proceso, México, Siglo xxı, 1977.

Friedmann, John, Empowerment. The politics of alternative development, Cambridge, Massachusetts, Blackwell, 1992.

González, Alma Amalia y Ronald Nigh, "Smallholder participation and certification of organic farm products in Mexico", en Journal of Rural Studies, núm. 4, vol. 21, 2005, pp. 449-460.

, Thierry Linck y Reyna Moguel, "El comercio de los valores éticos: las reglas del juego del café solidario", en Revista Europea de Estudios Latinoamericanos y del Caribe, vol. 75, 2003, pp. 31-46.

, "Ética y exclusión: las reglas del juego del mercado solidario de café", tesis doctoral, Toulouse, Université Toulouse Le-Mirail, San Cristóbal de las Casas, ECosur, 2003. - Evaluación de los beneficios actuales y el potencial para el combate a la pobreza de la participación en redes de comercio justo de café. Unión de Sociedades de La Selva, Federación de Sociedades de Solidaridad Social, Colorado, Fair trade research group, Colorado State University/Desarrollo Alternativo AC, 2002.
_Les règles du jeu du marché solidaire, Memoire du DEA ESSOR, Toulouse, ENFA Toulouse-Auzeville, ENSA Toulouse, Université Toulouse Le-Mirail, 1999.

Lipovetsky, Gilles, El crepúsculo del deber. $\mathrm{La}$ ética indolora de los nuevos tiempos democráticos, Barcelona, Anagrama, 2005.

Luhmann, Niklas, Confianza, México-Santiago de Chile, Editorial Antropos/Universidad Iberoamericana, 1996.

Mangematin, Vincent, "La confiance: un mode de coordination dont l'utilisation dépende de ses conditions de production", en Thuderoz et al. (editores), La Confiance. Approches économiques et sociologiques, París-Québec, Gaetan Morin Èditeur, 1999, pp. 31-56.

Moguel, Patricia y Víctor Toledo, "Biodiversity conservation in traditional coffee systems of Mexico" en Conservation Biology, núm. 1, vol. 13, 1999, pp. 11-21.

Muradian, Ronald y Wim Pelupessy, "Governing the Coffee chain. The role of voluntary regulatory systems", en World Development, núm. 12, vol. 33, 2005, pp. 2029-2044.

Murray, Douglas, Laura Raynolds y Peter Taylor, "The future of Fairtrade Coffee: dilemmas facing Latin America's small-scale producers", en Development in Practice, núm. 2, vol. 16, 2006, pp.197-192.

Nigh, Ronald, "Agriculture in the information age: The transnational ecology of corporate versus smallholder farming", en Urban Anthropology, núms. 3-4, vol. 28, 1999, pp. 1-46.

Pelupessy, Wim y Elisabeth van Tilburg, "El mercado solidario de café y el pequeño productor en Centroamérica", en M. Samper (editor), Crisis y perspectivas del café latinoamericano, San José, CR ICAFE: UNA, 1994, pp. 239-259.

Pouliquen, Kattell, Le commerce équitable, París, Editions Marabout, 2003.

Raynolds, Laura, Poverty Alleviation Through Participation in Fair Trade Coffee Networks: Existing Research and Critical Issues, Background Paper Prepared for Project Funded by the Community and Resource Development Program, Nueva York, The Ford Foundation, 2002. 
Renard, Marie-Christine, "Quality certification and power in fair trade", en Journal of Rural Studies, núm. 4, vol. 21, 2005, pp. 419-431.

Rice, Robert, "Noble goals and challenging terrain: organic and fair trade coffee movements in the global marketplace", en Journal of Agricultural and Environmental Ethics, núm. 1, vol. 14, 2001, pp. 39-66.

Roozen, Nico y Frans Van der Hoff, La aventura del comercio justo, México, El Atajo, 2002.
Speich, Nadine, "Le label, un instrument pour les petits producteurs du Sud", en C. Auroi y C. Schümperli Younossian (editores), Le commerce durable. Vers de plus justes pratiques commerciales entre le Nord et le Sud, Ginebra, IUED, 2001, pp. 283-300.

Tallontire,Anne, "Challenges facing Fair Trade: which way now?", en Small Enterprise Development, núm. 3, vol. 13, 2002, pp. 12-24.

Touraine, Alain, Producción de la sociedad, México, UNAM-IIS-IFAL, 1995. 